\title{
Epistaxis limits the performed activities of daily living in proportion to its severity: a cross-sectional survey among patients with hereditary haemorrhagic telangiectasia*
}

\author{
Masaki Hayama', Yohei Maeda', Takeshi Tsuda', Hitoshi Akazawa', \\ Ayaka Nakatani', Sho Obata', Kazuya Takeda',2, Hidenori Inohara' \\ ' Department of Otorhinolaryngology-Head and Neck Surgery, Osaka University Graduate School of Medicine, Suita, Osaka, \\ Japan \\ 2 Department of Otolaryngology, Kindai University Faculty of Medicine, Osakasayama, Osaka, Japan
}

Rhinology Online, Vol 4: 120 - 126, 2021 http://doi.org/10.4193/RHINOL/21.028

* Received for publication:

June 8, 2021

Accepted: July 29, 2021

Published: August xx, 2021

\begin{abstract}
Background: We examined the severity of epistaxis in patients with hereditary haemorrhagic telangiectasia (HHT) and its relationship with the performed activities of daily living.
\end{abstract}

Methodology: This cross-sectional survey included 36 patients with HHT in Japan. An anonymous questionnaire survey was conducted regarding the severity of epistaxis, the measures adopted to prevent epistaxis, and the limitations in the performed activities of daily living. The latter was assessed using a visual analogue scale (VAS). The correlation between epistaxis severity and the VAS score was analysed using Spearman's rank correlation coefficient.

Results: Of the 36 participants surveyed, $94.4 \%$ had $>1$ episode of epistaxis/week. The mean epistaxis severity score (ESS) was 4.3 (range, 0.9-8.4). Limitations in daily life, going out (within a day), meeting with others, eating with others, and going on overnight trips were positively correlated with the ESS. To prevent nosebleeds, $44.4 \%$ and $41.7 \%$ of the participants used medications and avoided drying their nasal cavities, respectively.

Conclusions: Epistaxis impacted the daily life of patients with HHT in proportion to its severity. Nonetheless, less than half of the patients used medications or took precautions. Hence, further educational activities should be considered for medical professionals and patients.

Key words: hereditary haemorrhagic telangiectasia; epistaxis; activities of daily living; quality of life; severity of illness index

\section{Introduction}

Hereditary haemorrhagic telangiectasia (HHT), as named by Hanes in 1909 (also known as Osler-Weber-Rendu or Osler's disease $\left.{ }^{(1-4)}\right)$, is a systemic disease characterised by telangiectasis and arteriovenous malformations in various organs. In the field of otorhinolaryngology, it is well known as the cause of intractable epistaxis ${ }^{(5)}$. It is diagnosed based on the Curaçao clinical diagnostic criteria ${ }^{(6)}$. $\mathrm{HHT}$ is an autosomal-dominant genetic disorder caused by mutations in the ENG and ACVRL1 genes, referred to as HHT1 and HHT2, respectively ${ }^{(5)}$. Although ENG and ACVRL1 mutations account for the majority of HHT cases, muta- tions in the SMAD4 gene are also related to HHT development ${ }^{(7)}$. In Japan, the prevalence of $\mathrm{HHT}$ is estimated to be one in $5,000-8,000$ individuals $^{(8)}$. Interestingly, approximately $99 \%$ of Japanese patients may experience recurrent epistaxis, which is the most common symptom ${ }^{(9)}$. As epistaxis is one of the major factors impairing the quality of life (QoL) of patients with $\mathrm{HHT}^{(10-}$

13), it is useful for physicians and patients themselves to ascertain the severity of epistaxis and the epistaxis severity score (ESS), as proposed by Hoag et al. ${ }^{(14)}$. According to previous work, patients with severe epistaxis based on the ESS are more likely to have affected Physical Component Summary and present significantly 

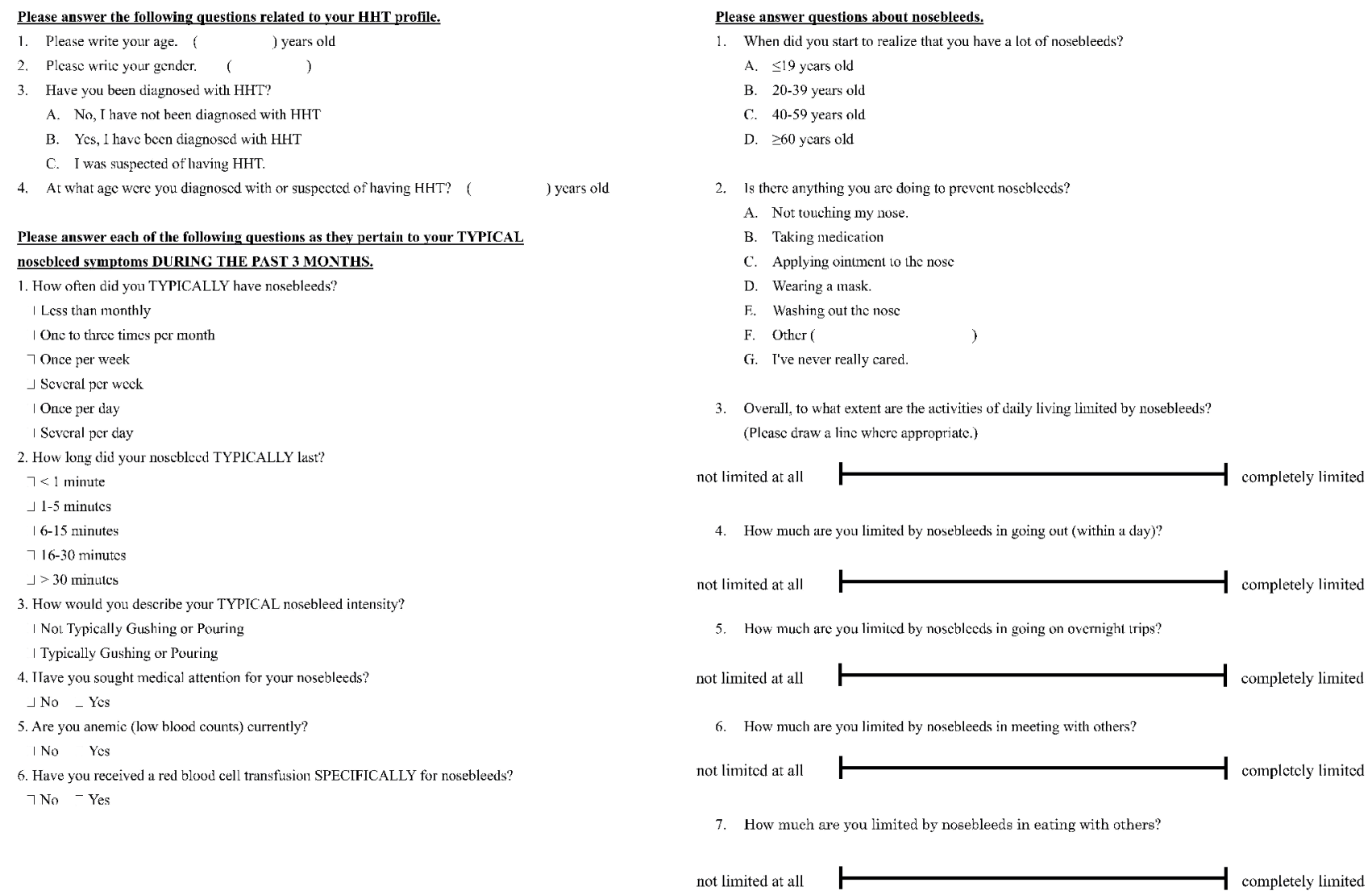

Figure 1. The questionnaire form regarding the ESS and the performed activities of daily living. This form was translated into English. The actual questionnaire was written in Japanese. ESS, epistaxis severity score.

worse Mental Component Summary scores than those with mild epistaxis $^{(13)}$. Arai and Akiyama reported that patients with HHT in Japan expected constant updates concerning HHT therapy, convenient access to HHT-specific epistaxis treatment, longer consultation time, acquisition of expert skills by the otolaryngologist, and sufficient knowledge to stop the recurrent nasal bleeding ${ }^{(15)}$. These expectations suggested that these issues had not been satisfied. Recurrent epistaxis may interfere with the patients' daily life. However, the extent to which epistaxis restricts their daily life and the type of treatment that patients with $\mathrm{HHT}$ receive have not been investigated. This information would aid medical professionals in gaining better insights into the patients condition and would help treatment guidance.

We hypothesised that HHT-related epistaxis limited the performed activities of daily living of these patients. It was also considered necessary to investigate the measures taken by patients with $\mathrm{HHT}$ to prevent epistaxis, as it would be beneficial for them to be informed if prevention was inadequate. Although the activities of daily living are an indicator of the patients' QoL, we aimed to investigate the restriction of the activity range more directly by conducting a questionnaire survey using the visual analogue scale (VAS). Therefore, with the cooperation of the Japan Osler's Disease Patient Association, we examined the severity of epistaxis and the correlation between epistaxis and daily behavioural restrictions to obtain knowledge on the severity of epistaxis among patients with $\mathrm{HHT}$ in Japan and highlight the importance of epistaxis prevention and treatment.

\section{Materials and methods}

\section{Questionnaire}

We conducted an anonymous questionnaire survey of patients with HHT who attended the 2017 annual meeting of the Japan Osler's Disease Patient Association. The questionnaire (Figure 1) was distributed at the meeting, and the patients were asked to complete it. We used the REDcapTM system as a questionnaire method for online responses. Patients who were unfamiliar with the use of the Internet were asked to fill out a paper questionnaire. A printed QR code form was distributed to those who wanted to complete the questionnaire online, and a questionnaire form was distributed to those who preferred a paperbased response. Both online and paper responses were received within 2 months. The survey items included age, sex, HHT diagnosis, the severity of epistaxis, and the degree of limitation in the activities of daily living.

Regarding the severity of epistaxis, the questions were focused 


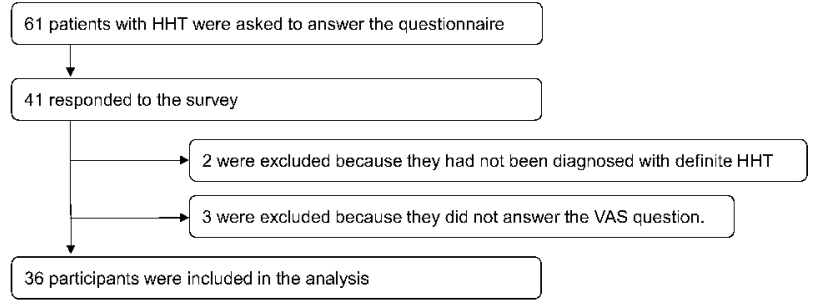

Figure 2. Flowchart of the survey participants. $\mathrm{HHT}$, hereditary haemorrhagic telangiectasia; VAS, visual analogue scale.

on the following: epistaxis frequency, duration, intensity, and history of epistaxis-related medical consultation, anaemia, and blood transfusion. The normalised ESS was calculated from the answers ${ }^{(14)}$. The impact on the activities of daily living was investigated using a 10-cm VAS in five categories: general daily life, going out (within a day), meeting with others, eating with others, and going on overnight trips. The participants were asked the following: 'How much of your daily social life is limited by epistaxis'? and 'To what extent are you restricted from going out (within a day)/meeting with others/eating with others/going on overnight trips because of epistaxis'? Preventive measures taken by the participants were also asked. The questions were primarily focused on the following issues: not touching my nose, taking medication, applying ointment to the nose, wearing a mask, washing out the nose, no care regarding the disease, and others. Multiple answers were allowed.
Table 1. Characteristics of the respondents.

\begin{tabular}{|lcc|}
\multicolumn{1}{c}{ Characteristic } & & \\
Sex & Male & $19(52.8 \%)$ \\
& Female & $17(47.2 \%)$ \\
Current age (years) & & $53.5(18-83)$ \\
\hline Age at HHT diagnosis (years) & & $42(6-70)$ \\
\hline Age of epistaxis onset (years) & $\leq 19$ & $17(47.2 \%)$ \\
& $20-39$ & $9(25 \%)$ \\
& $40-59$ & $10(27.8 \%)$ \\
& $\geq 60$ & $0(0 \%)$
\end{tabular}

Data are presented as numbers (percentages) or medians (ranges). $\mathrm{HHT}$, hereditary haemorrhagic telangiectasia.

\section{Statistical analysis}

All statistical analyses were performed using JMP Pro 14 Software (SAS Institute, Cary, NC). The correlation between the ESS and the degree of limitation in the activities of daily living was analysed using Spearman's rank correlation coefficient. The Kruskal-Wallis test was used to compare the limitations in the activities of daily living according to the ESS. For all tests, the level of significance was set at $p<0.05$.

\section{Ethics}

This study was approved by the Ethics Committee of Osaka University Hospital (\#17092). All participants provided written informed consent.

Table 2. Characteristics of epistaxis and normalised ESS.

\begin{tabular}{|c|c|c|c|}
\hline Characteristic & & $\mathbf{N}$ & $(\%)$ \\
\hline \multirow[t]{6}{*}{ Frequency of epistaxis } & Less than monthly & 0 & $0.0 \%$ \\
\hline & Once per month & 2 & $5.6 \%$ \\
\hline & Once per week & 3 & $8.3 \%$ \\
\hline & Several times per week & 8 & $22.2 \%$ \\
\hline & Once per day & 9 & $25.0 \%$ \\
\hline & Several times each day & 14 & $38.9 \%$ \\
\hline \multirow[t]{5}{*}{ Duration of epistaxis } & $<1 \min$ & 2 & $5.6 \%$ \\
\hline & $1-5 \min$ & 14 & $38.9 \%$ \\
\hline & $6-15 \min$ & 12 & $33.3 \%$ \\
\hline & $16-30 \mathrm{~min}$ & 4 & $11.1 \%$ \\
\hline & $>30 \mathrm{~min}$ & 4 & $11.1 \%$ \\
\hline \multirow[t]{2}{*}{ How would you describe your typical nose bleeding intensity? } & Not typically gushing & 24 & $66.7 \%$ \\
\hline & Typically gushing or pouring & 12 & $33.3 \%$ \\
\hline \multirow[t]{2}{*}{ Have you ever sought medical attention for nose bleeding? } & No & 28 & $77.8 \%$ \\
\hline & Yes & 8 & $22.2 \%$ \\
\hline \multirow[t]{3}{*}{ Are you anaemic (low blood count) currently? } & No & 13 & $36.1 \%$ \\
\hline & Yes & 18 & $50.0 \%$ \\
\hline & I do not know & 5 & $13.9 \%$ \\
\hline \multirow{2}{*}{$\begin{array}{l}\text { Have you ever received a red blood cell transfusion specifically because of } \\
\text { nose bleeding? }\end{array}$} & No & 35 & $97.2 \%$ \\
\hline & Yes & 1 & $2.8 \%$ \\
\hline \multirow[t]{3}{*}{ ESS [median (range), 4.3 (1.0-8.4)] } & Severe & 3 & $8.3 \%$ \\
\hline & Moderate & 17 & $47.2 \%$ \\
\hline & Mild & 16 & $44.4 \%$ \\
\hline
\end{tabular}

ESS, epistaxis severity score. 
Table 3. Relationship between the epistaxis severity score and the performed activities of daily living.

\begin{tabular}{lll}
$\begin{array}{l}\text { How limited is the following activity due to } \\
\text { epistaxis? }\end{array}$ & rs & p-value \\
\hline General daily life & 0.59 & $<0.01$ \\
Going out & 0.53 & $<0.01$ \\
\hline Meeting others & 0.57 & $<0.01$ \\
\hline Eating with others & 0.58 & $<0.01$ \\
\hline Going on overnight trips & 0.64 & $<0.01$
\end{tabular}

rs was calculated by Spearman's rank correlation coefficient.

\section{Results}

In total, 200 sphenoidal sinuses of 100 human cadaver (43 men, 57 women; mean age: $81 \pm 11,3$ years) were investigated. Sellar, post-sellar, lateral and combined sinus pneumatization type occurred in 100 (50\%), 46 (23\%), 26 (13\%) and 28 (14\%) sides. 44 of the specimens showed on at least one side an OCR. 20 of them had a unilateral OCR whereas the remaining 24 samples showed a bilateral OCR. In total, an OCR occurred in 68 of 200 (34\%) sides. Significant differences in OCR presence were found between sinus pneumatization types $\left[\chi^{2}(3)=20.09 ; p<0.001\right.$; $\mathrm{V}=0.317]$ : the combined type showed the highest prevalence of OCR presence $(14 / 28,50 \%)$, followed by lateral $(6 / 26,23 \%)$, post-sellar $(8 / 28,17 \%)$ and sellar $(12 / 100,12 \%)$, respectively. Divided according to the extent of pneumatization, a suboptical and latero-optical OCR were found in 19\% and 15\% of the sides (38/200 and 30/200), respectively. Detailed results of OCR subtypes according to sphenoid sinus pneumatization are presented in Table 1.

A bony dehiscence in the OCR area could be identified in 40 of 200 sides. Of the 61 patients with HHT who attended the 2017 annual meeting of the Japan Osler's Disease Patient Association, 41 participated in the survey (Figure 2). Among them, 39 parti- cipants answered that they had been diagnosed with definite HHT. Three participants who did not answer the VAS questions were excluded; thus, 36 patients were finally included in the analysis.

The respondents were 19 men and 17 women with a median age of 53.5 (range, 17-83) years (Table 1). All respondents reported $>1$ episode of epistaxis per month, and 34 (94.4\%) patients reported $>1$ episode per week. In total, 23 patients (63.9\%) suffered from daily epistaxis. The median ESS was 4.3 (range, 0.9-8.4). There were three severe, 17 moderate, and 16 mild cases of epistaxis (Table 2).

Table 3 shows the correlation between the degree of limitation in the activities of daily living and the ESS. The overall daily activities, going out, meeting people, eating with others, and the extent of travel restrictions for overnight trips had a significant correlation with the ESS (range, 0.53-0.64). When we compared the degree of limitation in the activities of daily living according to the epistaxis severity, the patients in the more severe group showed significantly greater limitations in the performed activities of daily living (Figure 3).

In the survey on measures taken to prevent epistaxis by themselves, most respondents (77.8\%) answered that they did not touch their noses (Table 4); $41.7 \%, 25.0 \%$, and $2.8 \%$ of the participants used an ointment, a mask, and performed nasal irrigation, respectively; and a total of $44.4 \%$ took preventive measures to prevent dryness of the nasal cavity. In the mild ESS group, only $18.8 \%$ of the participants used ointments, and $12.5 \%$ performed nasal irrigation. In contrast, a significantly greater proportion (58.8\%) applied ointment and nasal irrigation in the moderate ESS group ( $p<0.05$ ). In addition, $41.7 \%$ of the respondents answered that they had received a type of medication, while $13.9 \%$ answered that they did not take any special preventive measures.

Table 4. Preventive measures to avoid epistaxis daily, as reported by the patients (multiple answers were allowed).

\begin{tabular}{|c|c|c|c|c|c|c|}
\hline & \multicolumn{2}{|c|}{ Overall } & \multicolumn{2}{|c|}{ Mild } & \multicolumn{2}{|c|}{ Moderate } \\
\hline & $\mathbf{N}$ & Ratio & $\mathbf{N}$ & Ratio & $\mathbf{N}$ & Ratio \\
\hline Not touching my nose & 28 & $77.8 \%$ & 11 & $68.8 \%$ & 14 & $82.4 \%$ \\
\hline Taking medication & 9 & $25.0 \%$ & 3 & $18.8 \%$ & 3 & $17.6 \%$ \\
\hline Applying ointment to the nose & 15 & $41.7 \%$ & 3 & $18.8 \%$ & 10 & $58.8 \%$ \\
\hline Wearing a mask & 1 & $2.8 \%$ & 0 & $0.0 \%$ & 1 & $5.9 \%$ \\
\hline Washing out the nose & 15 & $41.7 \%$ & 2 & $12.5 \%$ & 10 & $58.8 \%$ \\
\hline Others & 7 & $19.4 \%$ & 4 & $25.0 \%$ & 2 & $11.8 \%$ \\
\hline No care regarding the disease & 5 & $13.9 \%$ & 4 & $25.0 \%$ & 1 & $5.9 \%$ \\
\hline
\end{tabular}

* Statistically significant $(p<0.05)$ (moderate vs mild). 

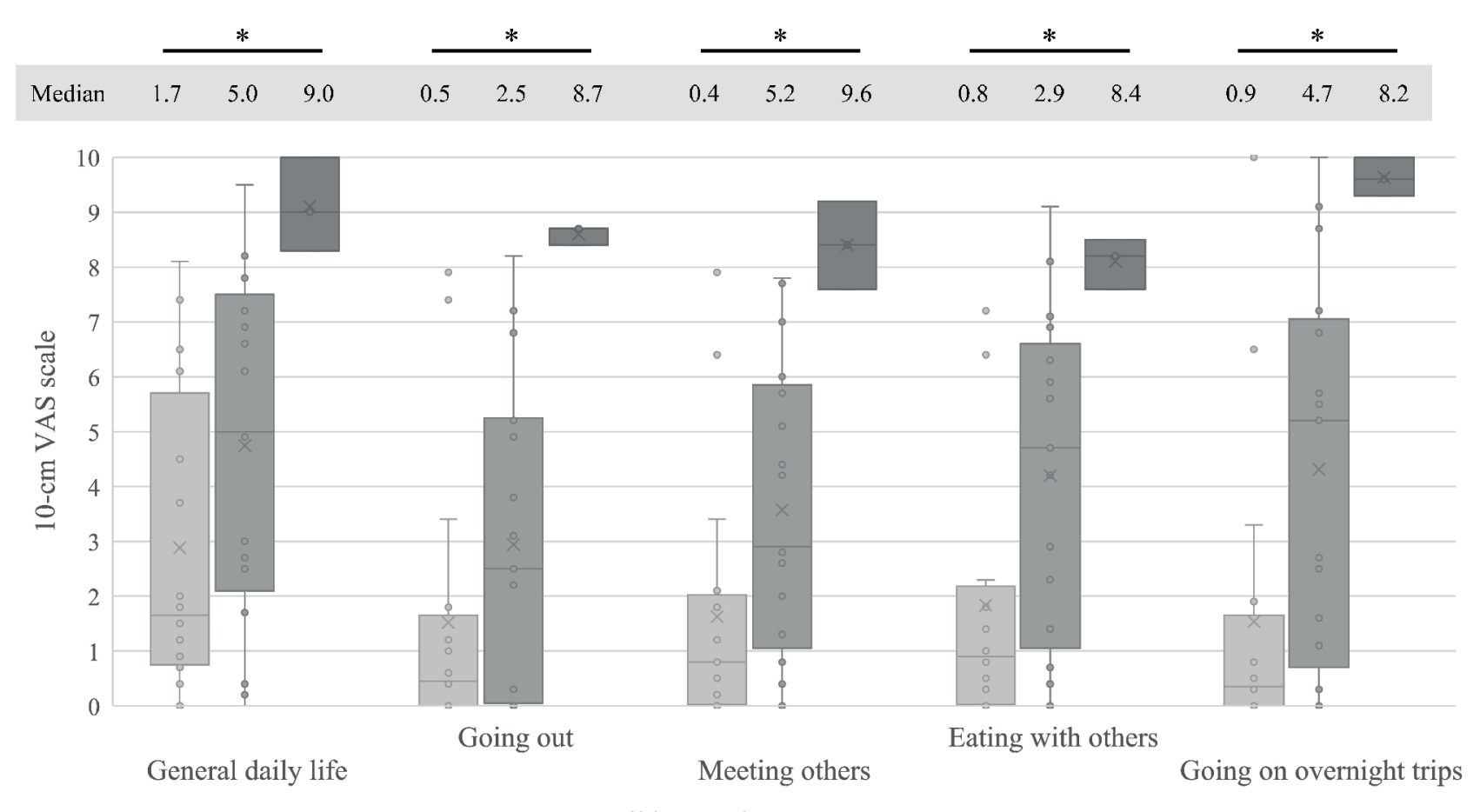

$\square$ mild $\square$ moderate $\square$ severe

Figure 3. Limitations in the activities of daily living by the severity of epistaxis ( $10 \mathrm{~cm}$-VAS scale). Each box shows the inter-quarter range. The median VAS score $(\mathrm{cm})$ for the range of actions in each group is presented at the top of the diagram. VAS, visual analogue scale. Comparison of each group was performed using ANOVA.

\section{Discussion}

In the present study, we investigated the severity of epistaxis and the correlation between epistaxis and daily behavioural restrictions among patients with HHT in Japan. Most respondents reported $>1$ episode of epistaxis per week. All activities of daily living were significantly correlated with the ESS. Moreover, patients with severe epistaxis showed significantly greater limitations in the activities of daily living than those with mild and moderate epistaxis. Furthermore, almost one-fifth of the patients answered that they did not take any special preventive measures.

The prevalence of epistaxis in HHT was reported to be 95$99 \%(9,14,15)$. In our study, the prevalence was $94.4 \%$, which was consistent with the results of having $>1$ episode of epistaxis per week. Gonzalez et al. reported that the severity of epistaxis was mild in children, even in confirmed HHT cases $^{(16)}$. Nonetheless, $50 \%$ of patients reported the presence of epistaxis by the age of 20 years, which was consistent with our finding that $47.2 \%$ of patients had recurrent epistaxis by the age of 19 years. These findings suggested that most patients with HHT have recurrent epistaxis as a part of their lives.

In this study, we found that the ESS was correlated with the limitations in the activities of daily living in patients with HHT. Merlo et al. reported that the ESS is a major determinant of impaired health-related QoL (HR-QoL) in these patients ${ }^{(13)}$. Geisthoff et al. stated that the duration of epistaxis, as well as liver involvement, gastrointestinal bleeding, and the number of visible telangiectases, presented a major influence on the HR-QoL of patients with $\mathrm{HHT}^{(10)}$. The management of recurrent epistaxis may be effective for improving the QoL of these patients and for preventing anaemia.

Drug therapy for epistaxis has been tested. In a phase IIIB, randomised, double-blind, placebo-controlled, cross-over study, treatment with tranexamic acid resulted in a decreased ESS by $54 \%{ }^{(17)}$. Gaillard et al. also demonstrated a $17 \%$ reduction in the mean duration of epistaxis per month in a controlled randomised, double-blind study ${ }^{(18)}$. Topical administration of antivascular endothelial growth factor antibodies, bevacizumab, and 5-fluorouracil has been attempted, but these therapies have not been well established ${ }^{(19-21)}$. The French Society of Otorhinolaryngology recommends the administration of tranexamic acid if there are no cardiovascular contraindications ${ }^{(22)}$. However, in our survey, only $41.7 \%$ of the patients received medication, suggesting the necessity of educational activities for medical professionals. These results may indicate why patients with HHT expect expertise from otolaryngologists ${ }^{(15)}$.

Regarding epistaxis prevention, avoiding nasal dryness and crusting may prevent the triggering of bleeding ${ }^{(23)}$. In a British study, in which a questionnaire survey was conducted in patients with $\mathrm{HHT}$, room humidification, nasal lubrication, and 
saline treatment were reported to be beneficial ${ }^{(24)}$. Therefore, at present, guidance to prevent nasal dryness and crust adhesion can prevent nasal bleeding. In the severe ESS group, all patients (100\%) answered that they prevented nasal dryness. However, only $58.8 \%$ of the participants stated that they had taken preventive measures in the moderate ESS group. Although this proportion is not low, it is not adequate. Despite the limitations caused by epistaxis in daily life activities, epistaxis prevention is not sufficiently widespread. Given the lack of patient guidance and the inadequacy of drug therapy, otolaryngologists in Japan should be better educated regarding HHT.

This study had several limitations. First, the number of participants was small. Second, as it was a questionnaire survey, the diagnosis of HHT was self-reported, and information regarding cerebrovascular or pulmonary complications was not obtained. Third, only $8.3 \%$ of cases were severe; therefore, this study was primarily focused on mild and moderate cases. Fourth, our questionnaire was original and had not been validated. Further prospective studies are needed to determine whether controlled epistaxis can reduce the degree of daily life limitation.

\section{Conclusions}

Epistaxis impacted the daily life of patients with HHT in proportion to its severity. In this study, the cohort mainly consisted of cases with mild and moderate epistaxis. Nevertheless, we found that the group with moderate epistaxis, in particular, experienced significant limitations in daily life. In addition, less than half of the patients took medications or adopted precautionary measures. Hence, further educational activities should be considered for medical professionals and patients.

\section{List of abbreviations}

ESS, epistaxis severity score; $\mathrm{HHT}$, haemorrhagic telangiectasia; HR-QoL, health-related quality of life; QoL, quality of life; VAS, visual analogue scale.

\section{Authorship contribution}

$\mathrm{HI}$ and $\mathrm{MH}$ supervised the project. $\mathrm{YM}, \mathrm{TT}, \mathrm{HA}$, and $\mathrm{MH}$ analysed the data and wrote the manuscript. YM, TT, HA, AN, SO, YM, and $\mathrm{KT}$ provided advice on project planning and data interpretation. All authors participated in the discussion of the results and critically polished and approved the final draft.

\section{Acknowledgments}

This work was supported by JSPS KAKENHI Grant (no. 19K09890). We would like to thank Mr Tadahiro Murakami, Chairman of the Osler's Disease Patients Association and all members of the Patients Association for their participation in the survey. We would also like to thank Editage (www.editage.com) for English language editing.

\section{Funding}

This work was supported by a KAKENHI Grant-in-Aid for Scientific Research from the Japan Society for the Promotion of Science [grant number 19K09890]. The funding source had no role in the study design; in the collection, analysis, and interpretation of data; in the writing of the report; and in the decision to submit the article for publication.

\section{Ethics approval and consent to participate}

This report was approved by the Institutional Review Board of Osaka University Hospital.

\section{Consent for publication}

Written informed consent was obtained from all participants.

\section{Availability of data and materials}

The datasets used and/or analysed during the current study are available from the corresponding author on reasonable request.

\section{Conflict of interest}

The authors declare that they have no competing interests.

\section{References}

1. Hanes F. Multiple hereditary telangiectases causing hemorrhage (hereditary hemorrhagic telangiectasia). Bull Johns Hopkins Hosp 1909; 20: 63-73.

2. Osler W. On a family form of recurring epistaxis, associated with multiple telangiectases of the skin and mucous membranes. Bull Johns Hopkins Hosp 1901; 12: 333-337.

3. Rendu H. Repeated epistaxis in a subject with small cutaneous and mucosal angiomas [article in French]. Gaz Hop 1896; 135: 1322-1323.

4. Parkes Weber F. Multiple hereditary developmental angiomata (telangiectases) of the skin and mucous membranes associated with recurring haemorrhages. Lancet 1907;
170: 160-162.

5. Faughnan ME, Palda VA, Garcia-Tsao G, et al. International guidelines for the diagnosis and management of hereditary haemorrhagic telangiectasia. J Med Genet 2011; 48: 73-87.

6. Shovlin $C L$, Guttmacher $A E$, Buscarini $E$, et al. Diagnostic criteria for hereditary hemorrhagic telangiectasia (Rendu-Osler-Weber syndrome). Am J Med Genet 2000; 91: 66-67.

7. McDonald J, Wooderchak-Donahue W, VanSant WC, Whitehead K, Stevenson DA, Bayrak-Toydemir P. Hereditary hemorrhagic telangiectasia: genetics and molecular diagnostics in a new era. Front Genet 2015; 6: 1.

8. Dakeishi M, Shioya T, Wada Y, et al. Genetic epidemiology of hereditary hemorrhagic telangiectasia in a local community in the northern part of Japan. Hum Mutat 2002; 19: 140-148.

9. Komiyama M, Ishiguro T, Yamada O, Morisaki H, Morisaki T. Hereditary hemorrhagic telangiectasia in Japanese patients. J Hum Genet 2014; 59: 37-41.

10. Geisthoff UW, Heckmann K, D'Amelio R, et al. Health-related quality of life in hereditary hemorrhagic telangiectasia. Otolaryngol Head Neck Surg 2007; 136: 726-733.

11. Zarrabeitia R, Fariñas-Álvarez C, Santibáñez M, et al. Quality of life in patients with hereditary haemorrhagic telangiectasia (HHT). Health Qual Life Outcomes 2017; 15: 19.

12. Geirdal A $\varnothing$, Dheyauldeen S, BachmannHarildstad G, Heimdal K. Quality of life in 
patients with hereditary hemorrhagic telangiectasia in Norway: A population based study. Am J Med Genet A. 2012; 158: 12691278.

13. Merlo CA, Yin LX, Hoag JB, Mitchell SE, Reh DD. The effects of epistaxis on health-related quality of life in patients with hereditary hemorrhagic telangiectasia. Int Forum Allergy Rhinol 2014; 4: 921-925.

14. Hoag JB, Terry P, Mitchell S, Reh D, Merlo CA An epistaxis severity score for hereditary hemorrhagic telangiectasia. Laryngoscope 2010; 120: 838-843.

15. Arai N, Akiyama T. A questionnaire-based survey to evaluate and improve the current HHT medical and social condition in Japan. Surg Neurol Int 2020; 11: 323.

16. Gonzalez CD, Mcdonald J, Stevenson DA et al. Epistaxis in children and adolescents with hereditary hemorrhagic telangiectasia. Laryngoscope 2018; 128: 1714-1719.

17. Geisthoff UW, Seyfert UT, Kübler M, Bieg B, Plinkert PK, König J. Treatment of epistaxis in hereditary hemorrhagic telangiectasia with tranexamic acid-a double-blind placebo-controlled cross-over phase IIIB study. Thromb Res 2014; 134: 565-571.

18. Gaillard S, Dupuis-Girod S, Boutitie F, et al. Tranexamic acid for epistaxis in hereditary hemorrhagic telangiectasia patients: A
European cross-over controlled trial in a rare disease. J Thromb Haemost 2014; 12: 1494-1502.

19. Whitehead KJ, Sautter NB, McWilliams JP, et al. Effect of topical intranasal therapy on epistaxis frequency in patients with hereditary hemorrhagic telangiectasia: a randomized clinical trial. JAMA 2016; 316: 943-951.

20. Dupuis-Girod S, Ambrun A, Decullier E, et al. Effect of bevacizumab nasal spray on epistaxis duration in hereditary hemorrhagic telangectasia: A randomized clinical trial. JAMA 2016; 316: 934-942.

21. de Jel DVC, Disch FJM, Kroon S, Mager JJ, Verdam FJ. Intranasal Efudix reduces epistaxis in hereditary hemorrhagic telangiectasia. Angiogenesis 2020; 23: 271-274.

22. Robard L, Michel J, Prulière Escabasse $V_{1}$ et al. Guidelines of the French Society of Otorhinolaryngology (SFORL) (short version). Specific treatment of epistaxis in Rendu-Osler-Weber disease. Eur Ann Otorhinolaryngol Head Neck Dis 2017; 134: 37-41.

23. Lupa MD, Wise SK. Comprehensive management of hereditary hemorrhagic telangiectasia. Curr Opin Otolaryngol Head Neck Surg 2017; 25: 64-68.

24. Silva BM, Hosman AE, Devlin HL, Shovlin CL.
Lifestyle and dietary influences on nosebleed severity in hereditary hemorrhagic telangiectasia. Laryngoscope 2013; 123: 1092-1099.

\section{Masaki Hayama, MD, PhD \\ Assistant Professor \\ Department of Otorhinolaryngology- \\ -Head and Neck Surgery \\ Osaka University Graduate School of \\ Medicine \\ 2-2 Yamada-oka \\ Suita \\ Osaka 565-0871 \\ Japan}

Tel: +81-6-6879-3951

Fax: +81-6-6879-3959

E-mail:

mhayama@ent.med.osaka-u.ac.jp

ISSN: 2589-5613 / @2021 The Author(s). This work is licensed under a Creative Commons Attribution 4.0 International License. The images or other third party material in this article are included in the article's Creative Commons license, unless indicated otherwise in the credit line; if the material is not included under the Creative Commons license, users will need to obtain permission from the license holder to reproduce the material. To view a copy of this license, visit http://creativecommons.org/ licenses/by/4.0/ 